\title{
The role of noncovalent interactions in the properties of porous compounds
}

\section{Catharine Esterhuysen}

\author{
Department of Chemistry and Polymer Science, Stellenbosch University, Matieland, Stellenbosch, 7602, South Africa \\ ce@sun.ac.za
}

Noncovalent interactions play a fundamentally important role in the properties of solid materials. For instance, guests are taken up into the host framework of porous materials as a result of the interactions between these species, while the manner in which they interact has an influence on the sorption ability of the porous material. In this work calculations on a range of porous frameworks allow us to explain the role that noncovalent interactions play in the sorption properties of these compounds. For instance, the origin of anomalous sorption isotherms are shown to be the result of interactions between acetylene ${ }^{[1]}$ or carbon dioxide ${ }^{[1,2]}$ and the host frameworks, as well as interactions between guests. Similarly, noncovalent interactions are responsible for the change in colour along an hourglass pattern of a crystalline porous compound during sorption of particular solvents. Calculations show that the origin of this effect is that the channels in the porous framework are anisotropic, allowing sorption only from particular faces. ${ }^{[3]}$

1
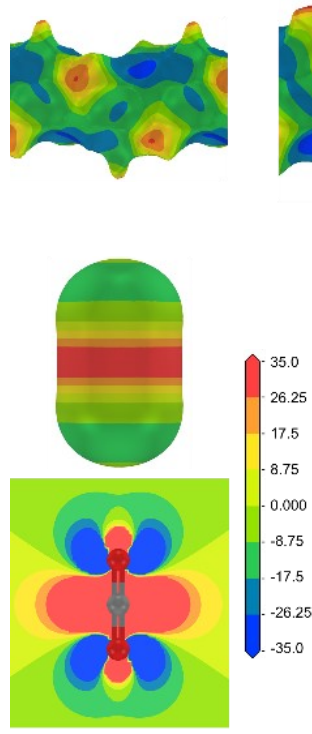

$\mathrm{CO}_{2}$ MEP
2
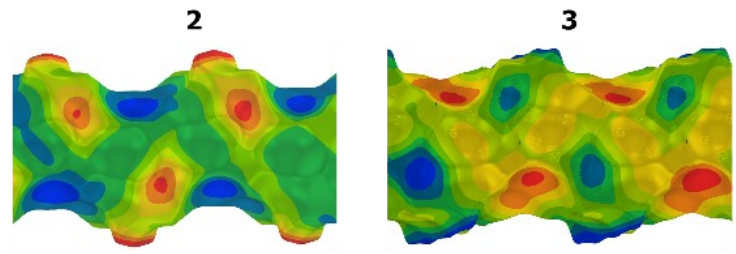

1

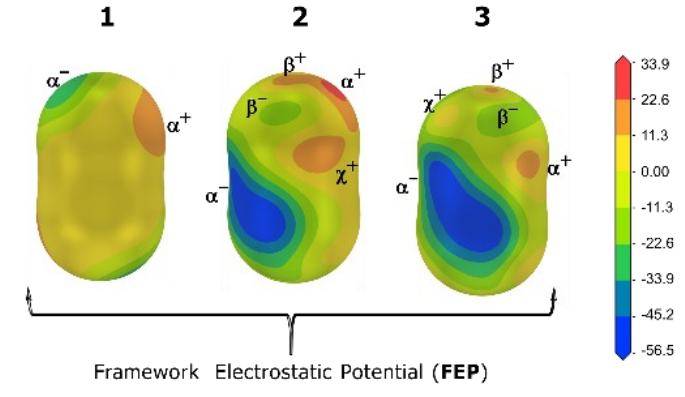

Framework Electrostatic Potential (FEP)

[1] Jacobs, T.; Lloyd, G. O.; Gertenbach, J. A.; Esterhuysen, C.; Müller-Nedebock, K. K.; Barbour, L. J., Angew. Chem. Int. Ed., 2012, 51, 49134916.

[2] Bezuidenhout, C. X.; Smith, V. J.; Bhatt, P. M.; Esterhuysen, C.; Barbour, L. J., Angew. Chem. Int. Ed. 2015, 54, $2079-2083$.

[3] Bezuidenhout, C. X.; Esterhuysen, C.; Barbour, L. J., Chem. Commun., 2017, 53, 5618-5621.

Keywords: Porous compounds, Computational chemistry, Noncovalent interactions 